\title{
A TOPOLOGY FOR AUTOMATA II
}

\author{
ARUN K. SRIVASTAVA \\ Department of Mathematics \\ Banaras Hindu University \\ Varansi, India \\ and \\ WAGISH SHUKLA \\ Department of Mathematics \\ Indian Institute of Technology \\ New Delhi, India \\ (Received October 6, 1983)
}

ABSTRACT. A topology on the state set of an automaton is considered and it is shown that under this topology, genetically closed subsets and primaries, in the sense of Bavel [1] turn out to be precisely the regular closed subsets and minimal regular closed subsets respectively. The concept of a compact automaton is introduced and it is indicated that it can be viewed as a generalization of a finite automaton. Included also is an observation showing that our topological considerations can help recover some of the results of Dörfler [2].

KEY WORDS AND PHRASES. Automata, topological concepts and methods, compact automata, product automata, connectedness.

1980 MATHEMATICS SUBJECT CLASSIFICATION CODE. 68D

1. INTRODUCTION.

Shukla and Srivastava [3] have indicated that it is possible to study certain aspects of automata e.g., the connectivity and separation properties, by using topological concepts and methods. They did this by introducing a topology on the state set of an automaton in a natural way. In this note, we consider a topology dual to this and show that a genetically closed set and hence a primary of an automaton have standard topological analogs. We also introduce the concept of a compact automaton and show that it can be viewed as a generalization of a finite automaton. Included also are a few remarks concerning certain preservation properties of product automata.

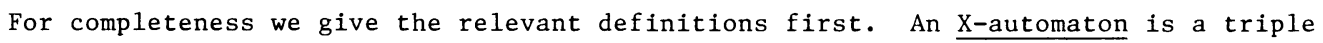
$A=(Q, X, \delta)$ where $Q$ is a set (set of states), $X$ is a semigroup (the $\underline{\text { input }}$ alphabet) with identity $e$, and $\delta: Q x x \rightarrow Q$ is a mapping (the transition map) satisfying for all $(\mathrm{q}, \mathrm{x}, \mathrm{y}) \varepsilon \mathrm{C} \times \mathrm{X} \times \mathrm{x}, \delta(\mathrm{q}, \mathrm{e})=\mathrm{q}$ and $\delta(\mathrm{q}, \mathrm{xy})=\delta(\delta(\mathrm{q}, \mathrm{x}), \mathrm{y}) \cdot \mathrm{B}=\left(\mathrm{Q}^{\prime} \mathrm{X}, \delta^{\prime}\right)$ is a subautomaton of $A$ iff $Q^{\prime} \subseteq Q$ and $\delta^{\prime}$ is $\delta$ restricted to $Q^{\prime} x X$. (We shall use $\delta$ for its restriction $\delta^{\prime}$ when no ambiguity arises). For a subset $R \subseteq Q$, the set $\delta R=\{\delta(q, x) \mid(q, x) \varepsilon R \quad x\}$ is the set of successors of $R, \sigma R=\{q \varepsilon Q \mid \delta(q, x) \varepsilon R$ for some $x \varepsilon X\}$ is the set of sources of $R$ and $\mu R=\{q \varepsilon R \mid \sigma q \subseteq R\}$ is the core of $R$. (We write $\sigma q$ for $\sigma\{q\}$ ). If $A=(Q, X, \delta)$ and 
$B=(P, X, \gamma)$ are $X$-automata then $f$ is a homomorphism from $A$ to $B$ if $f: Q+P$ is a function such that $f(\delta(q, x))=\gamma(f q, x)$ for $a 11(q, x) \in Q x X$.

If $A=(Q, X, \delta)$ is an $X$-automaton, then a topology $t A$ on $A$ can be defined by declaring a subset $R$ of $Q$ closed iff $R=\sigma R$ (cf.[3]). It turns out that

THEOREM 1.1([3]). If $B=\left(Q^{\prime}, X, \delta\right)$ and $A=(Q, X, \delta)$ are automata then

(a) $B$ is a subautomaton of $A$ iff $Q^{\prime}$ is tA-open.

(b) $B$ is a separated subautomaton of $A$ iff $Q^{\prime}$ is both tA-open and tA-closed.

(c) A is connected iff tA is connected (in topological sense).

(d) B is a block of $A$ iff $Q^{\prime}$ is a component of $Q$.

(e) $A$ is retrievable iff $t A$ is an $R_{0}$-topology.

(f) A is strongly connected iff tA is indiscrete.

Henceforth, in the absence of any other specifications, we shall assume $Q$ to carry the topology tA; this topology will be referred to as the 'state-set topology'.

A11 undefined concepts used here are either standard or can be found in Shukla and Srivastava [3] and the references cited therein.

2. CORE OPERATOR.

The core operator, as a self-map on the set of subsets of $Q$, turns out to be an interior operator in topological sense, i.e., the core operator satisfies

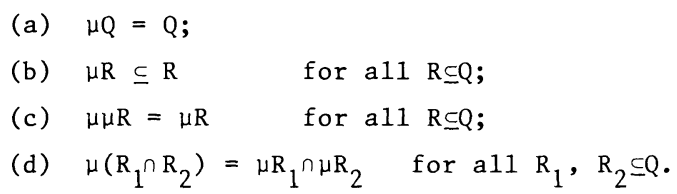

Indeed, (a) and (b) are obvious. To see (c), let $q \varepsilon \mu R$. Observe that $q \varepsilon \mu \mu R$ means that $\sigma_{q} \subseteq \mu R_{w h i c h}$ in turn means that for all $q \varepsilon Q$ such that $\delta(p, x)=q$ (for some $x \in X$ ), it is the case that $\sigma P \subseteq R$. So let $r \varepsilon Q$ be such that $\delta(r, y)=p$ for some $y \in X$. Then $\delta(r, y x)=q$ and so $r \varepsilon \sigma q$. Since $q \varepsilon \mu R, r \varepsilon R$. Thus, $\mu R \subseteq \mu \mu R$. But owing to (b), we also see that $\mu \mu R \subseteq \mu R$. Hence $\mu R=\mu \mu R$ and (c) is true. Finally, to verify (d), let $q \varepsilon \mu\left(R_{1} \cap R_{2}\right)$. Then $\sigma q \subseteq R_{1} \cap R_{2}$ whence $\mu\left(R_{1} \cap R_{2}\right) \subseteq \mu R_{1} \cap \mu R_{2}$. Conversely, if $q \varepsilon \mu R_{1} \cap \mu R_{2}$ then $\sigma q \subseteq R_{1}$ and $\sigma q \subseteq R_{2}$. It follows that (d) is also true.

The core, being an interior operator for $Q$, defines a topology on $Q$; a subset $R \subseteq Q$ is open with respect to this topology iff $R=\mu R$.

REMARK 2.1. (a) The above topology is saturated in the sense that any intersection of its open sets is open. To see this let $\left\{R_{i}: i \varepsilon I\right\}$ be any collection of its open sets. Then $R_{i}=\mu R_{i}$, for all $i \varepsilon I$. It is enough to show that $i \varepsilon I R_{i} \subseteq \mu\left(\hat{\ell}_{i} I R_{i}\right)$. Let $q \varepsilon \varepsilon_{i \varepsilon I} R_{i}$. Then $q \varepsilon \mu R_{1}$, for all $i \varepsilon I$ which means that $\sigma q \subseteq R_{i}$, for all $i \varepsilon I$. Hence $\sigma q \subset_{i \varepsilon I} R_{i}$ showing that $q \varepsilon \mu\left({ }_{i \varepsilon I} R_{i}\right)$. (b) The topology induced on $Q$ by $\mu$ is dual to the topology $t A$ on $Q$ and for this reason we shall denote it by $t^{*}$. Moreover, with respect to the topology $t A, \mu R$ is the largest closed set contained in $R$ for any $R \subseteq Q$. Also, the successor operator $\delta$ is the closure operator for $t A^{*}$ and $\sigma R$ is the smallest open set containing $\mathrm{R}$.

Throughout by $Q^{*}$, we shall understand the topological space $\left(Q, t A^{*}\right)$.

3. REGULAR CLOSED SUBSETS.

A subset of a topological space is called regular closed iff it equals the closure of its interior. In Bavel [1] a subset $R \subseteq Q$ is called genetic iff $\sigma R \subseteq \delta R$, genetic for $M \equiv Q$ iff $R$ is genetic and $\delta R=M$, genetically closed iff there exists a subset $M \subseteq R$ such that $\sigma M \subseteq \delta M$ and $\delta M=R$, and primary iff $R$ is a minimal genetically closed set. 
PROPOSITION 3.1. $R \subseteq Q$ is genetically closed iff it is a regular closed subset of $Q^{*}$. PROOF. Suppose $R$ is genetically closed. Then, as shown in Bave1 [1], $\delta \mu R=R$, i.e., $R$ is a regular closed subset of $Q$. Conversely, let $R$ be a regularly closed subset of $Q$. Then $R=\delta \mu R$. From the definition of $\mu R$ it is clear that $\sigma \mu R \subseteq R=\delta \mu R$ which shows that $R$ is genetically closed.

COROLLARY 3.2. A primary of an automaton is precisely a minimal regular closed subset of Q*.

The above observation, aided by Theorem 4.1 of Bave1 [1], yields the following:

THEOREM 3.3. If $\mathrm{p} \in \mathrm{Q}$ then the following statements are equivalent:

(a) $\delta \mathrm{p}$ is a primary of $\mathrm{A}$;

(b) $\delta p$ is a regular closed subset of $Q^{*}$;

(c) $\{\mathrm{p}\}$ is not a nowhere dense subset of $Q^{*}$;

(d) $\delta p$ is a minimal regular closed subset of $Q^{*}$;

(e) $\mathrm{p} \varepsilon \mu \delta \mathrm{p}$

REl.AARKS. (a) Since closures of open sets are regular closed, it is clear that if $R \subseteq Q$, then $\delta \sigma R$, as noted in Bavel [1], is genetically closed. (b) It is known that a finite union of regular closed sets stays regular closed. However, under the operation of intersection, this need not be true. Genetically closed sets, thus, do not form closed sets of topology. It may however be noted that genetic sets which are also closed subsets of $Q^{*}$ constitute a topology on $Q$ for which they are precisely a11 the closed sets.

4. COMPACT AUTOMATA.

We call an automaton $\mathrm{A}=(\mathrm{Q}, \mathrm{X}, \delta)$ compact (Compact automata were called 'quasifinite' automata or 'finitely reachable' automata in Shukla and Srivastava [3].) iff tA is a compact topological space (we do not assume a compact space to be necessarily Hausdorff). It was pointed out in Shukla and Srivastava [3] that $Q$ is compact iff $Q *$ has a finite dense subset. This means that there is a finite subset $D$ of states of $Q$ which every other state of $Q$ can be reached.

Clearly, like finite automata, compact automata also have only a finite number of blocks. In the remaining section we shall observe that finite automata and compact automata are alike in a few more respects.

THEOREM 4.1. A primary of a compact automaton is necessarily a maximal singly generated sub-automaton.

This follows from a result of Bavel ([1], Corollary 4.7) coupled with the fact that compact automata are finitely generated. Thus structure-wise, there is no distinction between the primaries of a finite automaton and of a compact automaton. This paves way for formualting a few theorems on (infinite) compact automata which are analogs of theorems on finite automata. As examples, we give the following two theorems [of Bave 1 [4]].

THEOREM 4.2. (Primary Decomposition Theorem) Let $P_{1}, P_{2}, \ldots, P_{n}$ be the set of primaries of a compact automaton $(A, X, \delta)$ then

(i) $\quad \mathrm{A}=\mathfrak{i}_{\mathrm{U}=1}^{\mathrm{n}} \mathrm{P}_{\mathrm{i}}$

(ii) for any $i, 1 \leqq i \leqq n, A \neq \underset{i \neq j}{U} P_{i}$

For the next theorem, we need a notation due to Bavel [4]. If $A_{i}=\left(Q_{i}, X, \delta\right)$ and $A_{j}=\left(Q_{j}, X, \delta\right)$ are two subautomata of an automaton $A=(Q, X, \delta)$ and if $f_{i}: Q_{i} \rightarrow Q$ and $f_{j}: Q_{j} \rightarrow Q$, two functions such that they agree on $Q_{i} \cap Q_{j}$ then $f_{i} \vee f_{j}$ is defined as 
follows: $\left(f_{i} \vee f_{j}\right)(x)$ equals $f_{i}(x)$ if $x \in Q_{i}$ and it equals $f_{j}(x)$ if $x \varepsilon Q_{j}$.

THEOREM 4.3. (Homomorphism Decomposition Theorem). Let $A$ be a compact automaton and $B$ any other automaton. Let $P_{1}, P_{2}, \ldots, P_{n}$ be the primaries of $A$ and $f: A \rightarrow B$ be a homomorphism. Then there exist homomorphisms $f_{i}: P_{i} \rightarrow B, 1 \leqq i \leqq n$, such that $f$ has a 'decomposition' $f=\mathfrak{i = 1}_{i}^{n} f_{i}$. Furthermore, this decomposition is unique.

5. PRODUCTS OF AUTOMATA. Several definitions of products of automata are available in the literature. We consider here the categorical product of automata (in the category of automata and their homomorphisms) and observe that the state set topology functor from the category of automata to that of topological spaces preserves products. As a consequence certain observations made by Dorfler [2] are recovered.

The following definition of products of automata is easily extendible to any arbitrary family of automata. Let $A_{1}=\left(Q_{1}, X_{1}, \delta_{1}\right)$ and $A_{2}=\left(Q_{2}, X_{2}, \delta_{2}\right)$ be two automata. Then $A_{1} \times A_{2}=\left(Q_{1} \times Q_{2}, x_{1} \times x_{2}, \delta_{1} \times \delta_{2}\right)$, where $x_{1} \times x_{2}$ is the direct product of monoids $\mathrm{x}_{1}$ and $\mathrm{x}_{2}$ and $\delta_{1} \mathrm{x} \delta_{2}$ is defined by $\left(\delta_{1} \mathrm{x}_{2}\right)\left(\left(\mathrm{q}_{1}, \mathrm{q}_{2}\right),\left(\mathrm{x}_{1}, \mathrm{x}_{2}\right)\right)=\left(\delta_{1}\left(\mathrm{q}_{1}, \mathrm{x}_{1}\right), \delta_{2}\left(\mathrm{q}_{2}, \mathrm{x}_{2}\right)\right)$ for any $\left(\mathrm{q}_{1}, \mathrm{q}_{2}\right) \in \mathrm{Q}_{1} \times \mathrm{Q}_{2}$, and $\left(\mathrm{x}_{1}, \mathrm{x}_{2}\right) \in \mathrm{X}_{1} \mathrm{x}_{2}$ is an automaton and will be called the product of the automata $A_{1}$ and $A_{2}$. Of course this product is nothing but the usual parallel composition or the direct product in the sense of Dörfler [2]. It is easy to verify that it is also the categorical product.

THEOREM 5.1. Let $A_{1}=\left(Q_{1}, X_{1}, \delta_{1}\right)$ and $A_{2}=\left(Q_{2}, X_{2}, \delta_{2}\right)$ be two automata and $A_{1} \times A_{2}$ their product. Then $t A_{1} \times t A_{2}=t\left(A_{1} \times A_{2}\right)$, where $t A_{1} \times t A_{2}$ denotes the Tychonoff product of the state set topologies $t A_{1}$ and $t A_{2}$.

PROOF. First let $U$ be open in $t\left(A_{1} \times A_{2}\right)$. Then $\left(\delta_{1} x_{2} \delta_{2}\right) U=U$. Suppose $\left(\mathrm{q}_{1}, \mathrm{q}_{2}\right) \varepsilon \mathrm{U}$. Consider $\delta \mathrm{q}_{1} \mathrm{x} \delta \mathrm{q}_{2}$ which clearly is basic open in $t A_{1} \mathrm{xA}_{2}$. If $\left(\mathrm{q}_{1}^{\prime}, \mathrm{q}_{2}^{\prime}\right) \varepsilon \delta \mathrm{q}_{1} \times \delta \mathrm{q}_{2}$ then there exist $\mathrm{x}_{1} \varepsilon \mathrm{X}_{1}$ and $\mathrm{x}_{2} \varepsilon \mathrm{X}_{2}$ such that $\delta_{1}\left(\mathrm{q}_{1}, \mathrm{x}_{1}\right)=\mathrm{q}_{1}^{\prime}$ and $\delta_{2}\left(\mathrm{q}_{2}, \mathrm{x}_{2}\right)=\mathrm{q}_{2}^{\prime}$. Since $\left(\delta_{1} \mathrm{x} \delta_{2}\right) \mathrm{U}=\mathrm{U}$, it follows that $\left(\delta_{1} \mathrm{x}_{2}\right)\left(\left(\mathrm{q}_{1}, \mathrm{q}_{2}\right),\left(\mathrm{x}_{1}, \mathrm{x}_{2}\right)\right)$ $=\left(q_{1}^{\prime}, q_{2}^{\prime}\right) \varepsilon U$. Thus $U$ contains a basic open set (viz, $\left.\delta q_{1} x \delta q_{2}\right)$ around its each point $\left(\mathrm{q}_{1}, \mathrm{q}_{2}\right)$ and so must be open in $t A_{1} \times \mathrm{tA}_{2}$. Conversely, let $\mathrm{U}$ be open in $t A_{1} x \mathrm{~A}_{2}$ and let $\left(\mathrm{q}_{1}, \mathrm{q}_{2}\right) \in \mathrm{U}$. Then there must exist sets $\mathrm{U}_{1}$ and $\mathrm{U}_{2}$ open respectively in $t A_{1}$ and tA $A_{2}$ with $\left(q_{1}, q_{2}\right) \varepsilon U_{1} \times U_{2} \subseteq U$. Since $\delta_{1} U_{1}=U_{1}$ and $\delta_{2} U_{2}=U_{2}$, it follows that $\left(\delta_{1} \times \delta_{2}\right)$ $\left(q_{1}, q_{2}\right) \varepsilon U$ whence $\left(\delta_{1} \times \delta_{2}\right) U=U$ showing openness of $U$ in $t\left(A_{1} \times A_{2}\right)$.

It is known from topology that if $\mathrm{T}_{1}, \mathrm{~T}_{2}$ are two simultaneously indiscrete, connected, $R_{0}$ or compact topologies then so is their Tychonoff product and also conversely. Thus in view of Theorem 1.1, we have the following.

COROLLARY 5.2. Let $\mathrm{P}$ be any of the properties connectedness, strong connectedness, retrievability or compactness of an automaton. Let $A_{1}$ and $A_{2}$ be two antomata. Then $A_{1} \times A_{2}$ has the property $P$ iff both $A_{1}$ and $A_{2}$ have $P$.

The above observation in the case of $\mathrm{P}$ being connectedness or strong connectedness was made by Dörfler [2].

\section{REFERENCES}

1. BAVEL, Z. On the Decomposiibliity of Monadic Algebras and Automata, In. "Proceedings, Eighth Annual Symposium on Switching and Automata Theory, October 1967", (1967), 322-335.

2. DÖRFLR, W. The Direct Product of Automata and Quasiautomata, In. "Mathematical Foundation of Computer Science 1976", Lecture Notes in Computer Science No. 45, Springer-Verlag Berlin (1976), 270-276.

3. SHUKEA, W. and SRIVASTAVA, A.K. A Topology for Automata: A Note, Inform. Contr. 32, (1976), 163-168.

4. BAVEL, Z. Structure and Transition-preserving Functions of Finite Automata, J. Assoc. Comput. Mach. 15 (1968), 135-158. 


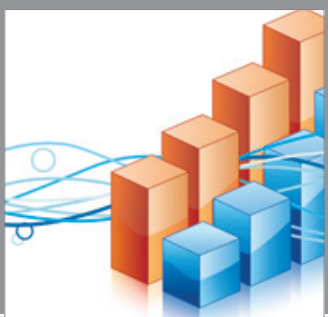

Advances in

Operations Research

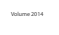

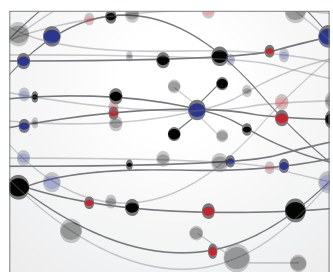

\section{The Scientific} World Journal
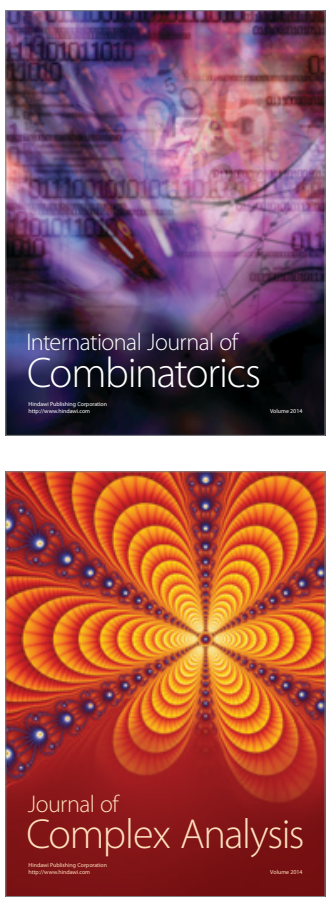

International Journal of

Mathematics and

Mathematical

Sciences
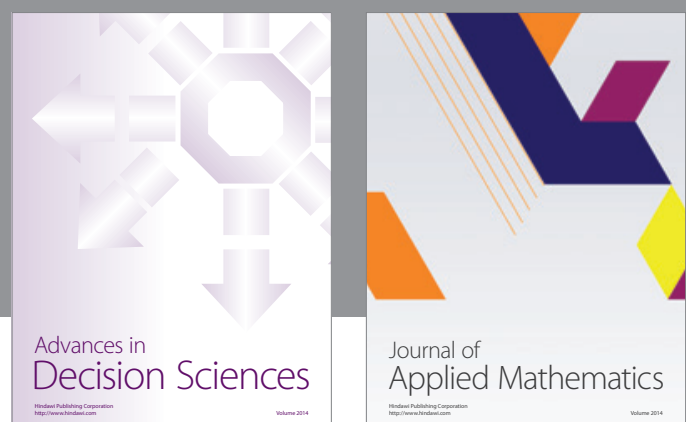

Journal of

Applied Mathematics
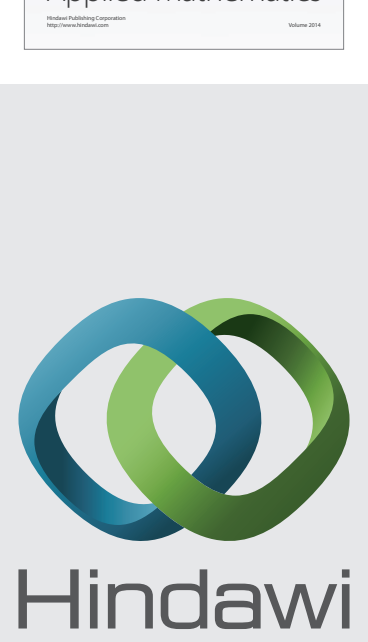

Submit your manuscripts at http://www.hindawi.com
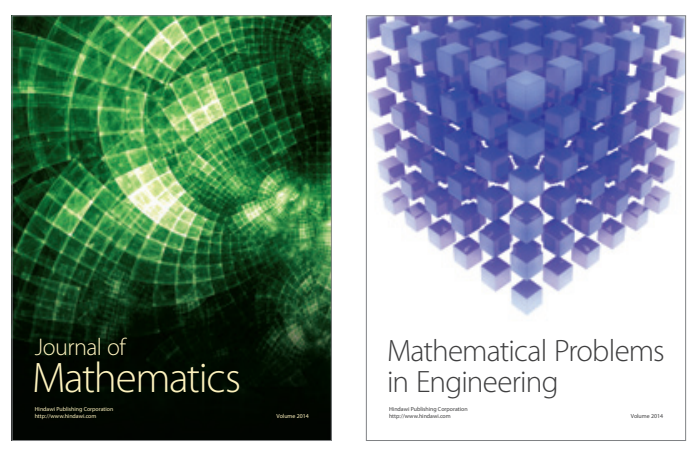

Mathematical Problems in Engineering
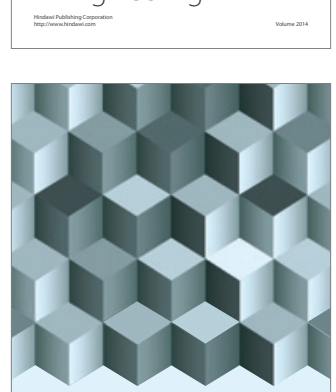

Journal of

Function Spaces
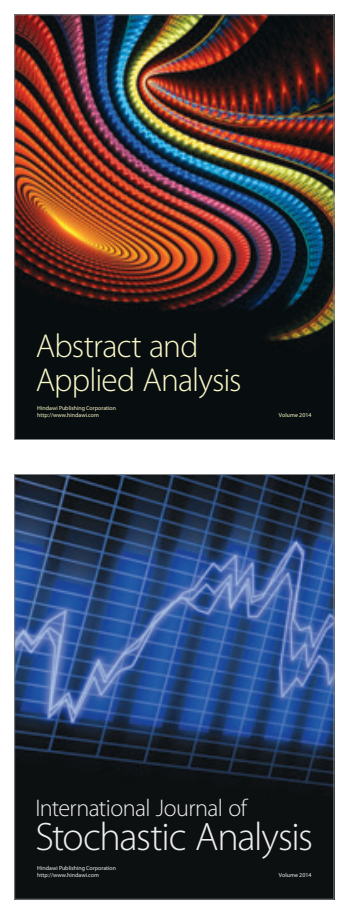

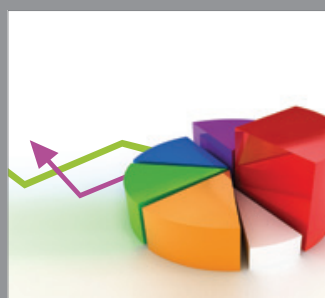

ournal of

Probability and Statistics

Promensencen
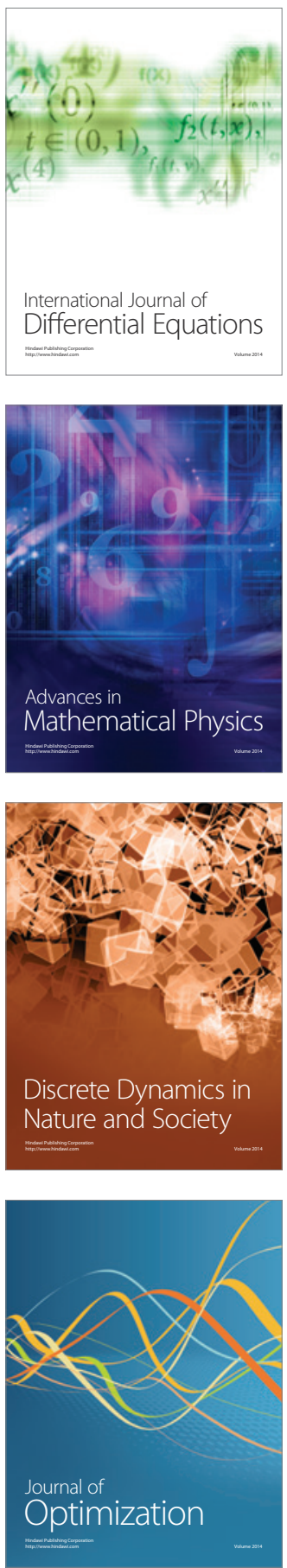